\title{
Contextual Influences of Trainee Characteristics and Daily Workload on Trainee Learning Preferences
}

\author{
Brita Roy, MD, MPH, MHS ${ }^{1 *}$, Nidhi Huff, $\mathrm{MD}^{2}$, Carlos Estrada, MD, MS ${ }^{3,4}$, Analia Castiglioni, $\mathrm{MD}^{5}$, \\ Lisa L. Willett, MD, MACM ${ }^{6}$, Robert M. Centor, MD $^{6,7}$
}

\begin{abstract}
'Section of General Internal Medicine, Yale School of Medicine, New Haven, Connecticut; ${ }^{2}$ Wellstar Pulmonary and Intensivist Group, Atlanta, Georgia; ' ${ }^{3}$ Division of General Internal Medicine, University of Alabama at Birmingham, Birmingham, Alabama; ${ }^{4}$ Birmingham Veterans Affairs Medical Center Quality Scholar Program, Birmingham, Alabama; ${ }^{5}$ University of Central Florida College of Medicine, Orlando, Florida; ${ }^{6}$ Division of General Internal Medicine, University of Alabama at Birmingham, Birmingham, Alabama.
\end{abstract}

\begin{abstract}
We previously defined teaching domains necessary for successful inpatient medicine attending rounds from the trainees' perspective in Role Modeling, Learning Environment, Teaching Process and Team Management. We sought to understand whether trainee characteristics and daily fluctuations in workload influence the prioritization of these domains. We conducted a prospective observational study in general medicine inpatient wards at a university, Veterans Affairs, and a county hospital affiliated with one academic institution over the course of 6 months. All student and resident trainees on internal medicine inpatient wards were eligible to participate. We designed a daily assessment tool on which trainees were asked to identify the teaching domain most important to them, along with information on sex, training level, call-cycle day, patient census, and number of team
\end{abstract}

members absent during rounds. We examined associations between training level and workload factors with the prioritized teaching domain using Pearson's chi-square analysis, adjusted for clustering effects. We collected 1,378 daily assessment cards evaluating 53 (91\%) attending physicians. Students valued Teaching Process $(P<0.001)$, while senior residents sought Team Management $(P<0.001)$. On most days, Teaching Process was prioritized $(P=0.005)$. On postcall days and days with a high patient census, Team Management was prioritized $(P<0.001)$. Attending physicians may consider tailoring rounds in response to work-related pressures. Days with a high workload are better suited for demonstrating efficient and effective patient care skills. Journal of Hospital Medicine 2017;12:558-561. (C) 2017 Society of Hospital Medicine
We previously identified key domains of attending attributes for successful rounds ${ }^{1}$ and adapted them to represent the trainees' perspective: Teaching Process (eg, sharing decision-making process, physical exam skills), Learning Environment (eg, being approachable, respectful), Role Modeling (eg, teaching by example, bedside manner), and Team Management (eg, efficiency, providing autonomy). Though all domains are necessary, the relative importance may change in response to external pressures. Inpatient service demands and time constraints fluctuate daily due to patient admissions and discharges, educational conference schedules, and concurrent outpatient clinic responsibilities. ${ }^{2-4}$ Furthermore, the 2011 Accreditation Council for Graduate Medical Education (ACGME) duty hour rules placed greater time pressure on inpatient ward attending rounds. ${ }^{5}$ It is plausible that these pressures affect trainees' needs and priorities during rounds.

\footnotetext{
*Address for correspondence and reprint requests: Brita Roy, MD, MPH, MHS, Section of General Internal Medicine, Yale School of Medicine, 367 Cedar St., ESH A, Suite 406A, New Haven, CT 06510; Telephone: 203-785-5564; Fax: 203-737-3306; E-mail: brita.roy@yale.edu

Additional Supporting Information may be found in the online version of this article.

Received: November 22, 2016; Revised: December 29, 2016; Accepted: January 1, 2017
}

2017 Society of Hospital Medicine DOI 10.12788/jhm.2771
Therefore, we sought to refine our understanding of the learners' needs during ward rounds. Because we were interested in the contextual influences of trainee characteristics and workload on their preferences, we used the principles of ecological momentary assessment (EMA) to design a novel system to assess daily changes in trainee priorities and associated workload.

\section{METHODS}

\section{Design, Participants, and Setting}

In a prospective observational study, we assessed trainee priorities during inpatient rounds. Participants included third- and fourth-year medical students in the University of Alabama at Birmingham (UAB) School of Medicine (Birmingham campus) and residents in the Tinsley Harrison Internal Medicine Residency Training Program assigned to inpatient general medicine ward services from September 2010 to February 2011 (except from December 20, 2010, to January 3, 2011). Three training sites were included in this study: UAB Hospital (>1000-bed, university-based hospital), Birmingham Veterans Affairs Medical Center (300bed), and Cooper Green Hospital (40-bed county hospital). Each site housed 4 or 5 general medicine ward teams, composed of 1-3 medical students (third or fourth year), 2 first-year residents, 1 upper level resident (postgraduate year 2-4), and 1 attending physician.

Up to 10 new patients were admitted to a team every 


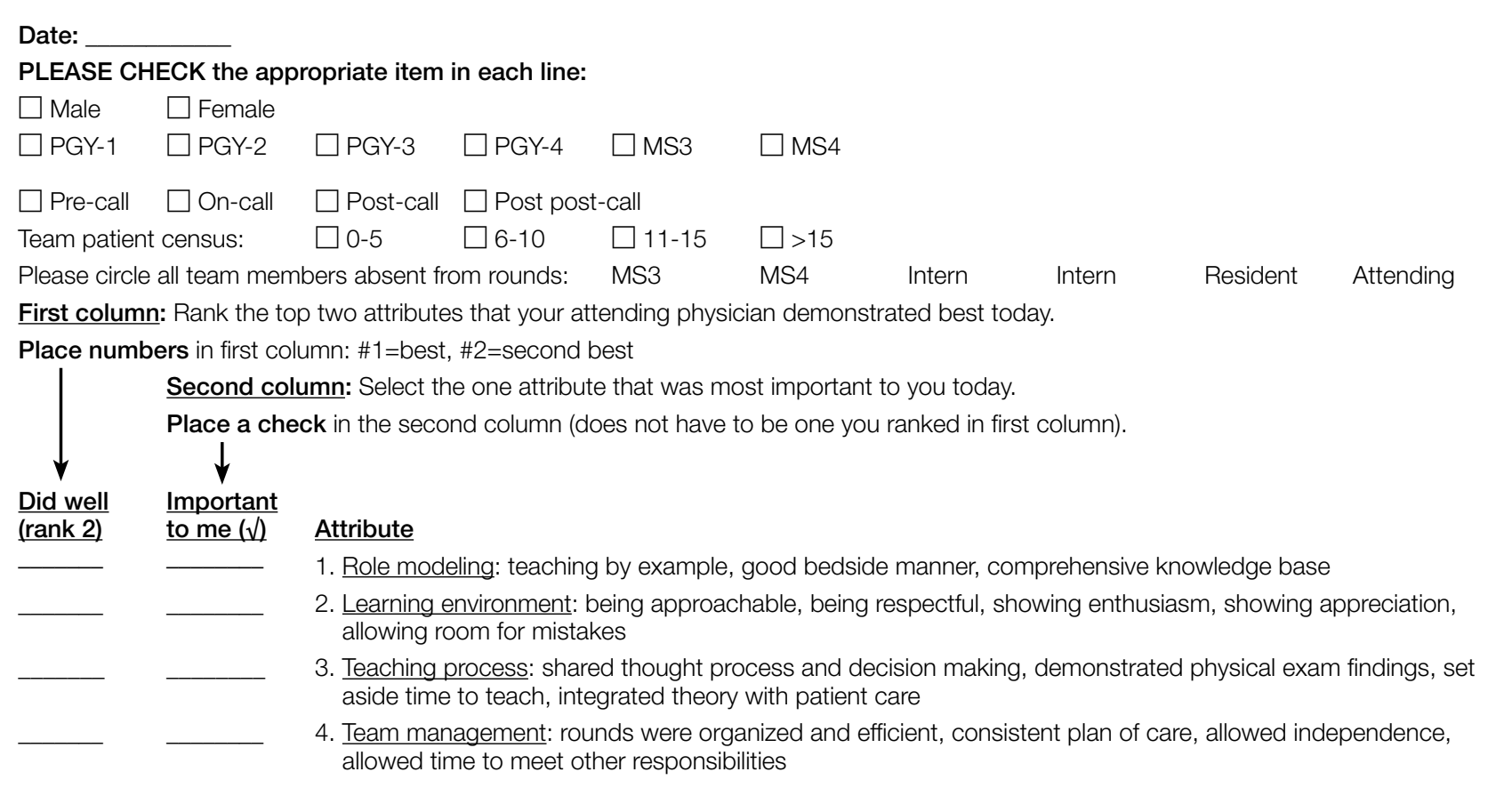

FIG 1. Ecological momentary assessment (EMA) card. Daily assessment tool created to determine which of 4 domains for successful ward rounds the trainee prioritized and factors impacting workload.

fourth day, the on-call day, at the Veterans and Cooper Green hospitals. At UAB hospital, up to 8 new patients were admitted to a team every fifth day with an additional 4 new patients 3 days after the on-call day. Typically, teams conducted daily rounds with the attending for up to 2 hours each morning. On post-call days, the day after the on-call day, rounds lasted approximately 3.5 hours. During on-call weekdays, upper level residents completed a 12-hour shift (7 Am to 7 PM). During on-call weekends, the upper level resident was responsible for admissions during a 24-hour period ( $7 \mathrm{Am}$ to $7 \mathrm{Am}$ ) and left the hospital before noon on the post-call day.

Trainees were recruited to participate via e-mail and verbal announcements during program conferences. Participation was voluntary and responses were confidential. As an incentive, the team submitting the highest number of cards per site each month received 1 free lunch. Institutional review boards of all 3 participating hospitals approved this study.

\section{Assessment}

Our original domains of attending rounds were derived from groupings and ratings of specific teaching characteristics by attending physicians, residents, and students. ${ }^{1}$ However, because our goal was to understand the learners' perspective of successful ward rounds, we revised these domains by limiting the algorithm to data on groupings and ratings by residents and students only. This process resulted in the domains used in this study (Appendix).

We used EMA principles to create a novel system to assess daily variation in trainees' prioritization of these domains and workload. ${ }^{6,7}$ EMA-derived assessment tools collect data frequently (several times per week, up to multiple times per day) to identify time-sensitive fluctuations. We designed a pocket-sized daily assessment card for trainees to complete each day after rounds (Figure 1). Trainees were asked to indicate the most important domain for successful ward rounds to them that day and provide individual characteristics (ie, sex and training level) and data on factors we hypothesized were related to perceived work load (ie, patient census, day of call cycle, and number of team members absent on rounds that day). We anticipated the Expectations domain would not be responsive to daily changes in workload because expectations are usually set once on the first day of the rotation, and thus we did not include this domain in our final assessment tool. Assessment cards and locked receptacles were kept in team workrooms for ease of accessibility; cards were collected twice monthly. All data were anonymous.

\section{Analysis}

Our unit of analysis was the EMA card. We examined associations between daily domain priority with respondent demographics and workload information using Pearson's chi-squared analyses, adjusted for clustering effects by team. $\alpha$ was set at 0.05 . All analyses were performed by using Stata 13.0 (Stata Statistical Software: Release 13; College Station, TX).

\section{RESULTS}

We collected 1,378 daily assessment cards over a 6-month period, with at least 1 participating member from 63 differ- 


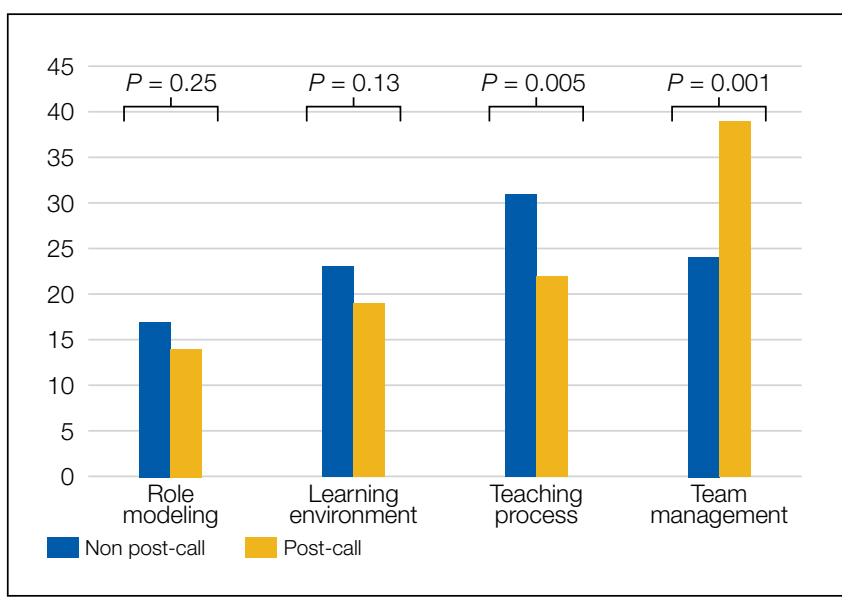

FIG 2. Domain priority by call-day. Percent of daily assessment cards with each domain selected as the trainees' top priority, stratified by post-call days vs all other days.

ent inpatient general medicine ward teams (81\%) led by 53 different attending physicians (91\%). Cards represented expected proportions across training levels according to the number of teams at each site and their composition during each rotation. Submission of EMA cards was well distributed across the study period. Overall, the 2 most important domains were Teaching Process (392/1378 cards; 31\%) and Team Management (370/1378 cards; 29\%).

Sex and training level were associated with prioritization of teaching domains. Male trainees were more likely to choose Team Management $(P=0.01)$ or Teaching Process $(P$ $=0.04)$ as their preferred domain. Medical students valued Teaching Process $42 \%$ of the time, compared with $23 \%$ for interns and $21 \%$ for upper level residents $(P=0.005)$. The opposite trend emerged for Team Management: as training level increased, the importance of Team Management increased $(P$ $<0.001)$. There were no significant trends by training level for the Role Modeling and Learning Environment domains.

Domain priority was also associated with workload characteristics. On post-call days, Team Management $(P<0.001)$ was more likely to be selected as the most important domain, but on other days, Teaching Process $(P=0.005)$ was more often selected (Figure 2). Trainees also selected Team Management as the most important domain with an increasing number of team members absent $(P=0.001)$, and as the teams' overall patient census increased $(P<0.001)$. The Learning Environment and Role Modeling domains' importance did not vary by call-day or patient census.

\section{DISCUSSION}

We used a novel approach to assess contextual factors affecting trainee prioritization of 4 domains that contribute to successful inpatient internal medicine attending rounds. We found training level and workload demands were associated with prioritization of teaching domains. Prioritization of Teaching Process, exemplified by setting aside time to teach, demonstrating physical exam skills, and clear delineation of the attending's thought process, was inversely associated with training level. On the days with highest workload, Team Management was most likely to be prioritized. Our findings suggest that attending physicians should consider adapting rounding style based on team members' training levels and workload.

Prior work has described teaching and rounding styles, influences, and priorities in response to workload from the attending physician's perspective, ${ }^{8-11}$ and our study extends these reports by providing the complementary perspective of trainees. On days with high workload, trainees prefer the Team Management domain, characterized by organized and efficient rounds, agreement on a clear and consistent plan of care, and being allowed independence and time during rounds to meet other responsibilities. ${ }^{1}$ These findings support an "empowerment style," defined by Goldszmidt et al. as using integrated teaching and oversight strategies to support trainees' progressive independence. ${ }^{9}$ Though some attending physicians report shifting to a more direct patient care style on days with a high patient census, ${ }^{9}$ our results suggest that learners instead prefer more independence, being empowered to perform more direct care. While there is an increasing pressure to heighten attending supervision due to concerns about patient safety, restricted work hours, and litigation, ${ }^{12}$ trainees value being part of the care process and being included as integral members of the care team, which may actually mitigate patient safety risks. ${ }^{8}$

Our results are consistent with prior studies, reporting that learners at different levels of training have different instructional needs: medical students seek more teaching, and senior residents sought an efficient leader. ${ }^{13,14}$ Taken together, these studies suggest that attending physicians should tailor rounds to the level of the trainee. For example, it may be beneficial for the attending physician to spend time outside of rounds with students to teach medical knowledge. During rounds, the entire group benefits most from modeling clinical reasoning, discussing new medical evidence, and demonstrating communication skills and leadership.

Our study has limitations. Though our study was performed before the 2011 ACGME duty hour restrictions, ${ }^{5}$ our results are likely of greater importance and relevance, as our findings ultimately highlight the competing demands of time vs duty. Also, while our study was performed at a single institution, potentially limiting generalizability, we included 3 types of training hospitals, a university, veterans and a county hospital, and found no differences between sites. Additionally, we collected over 1,000 cards over the course of 6 months, assessing rounds of over 50 different attending physicians, suggesting broader applicability. Our overall response rate was low, a typical signal for respondent bias, but because we collected daily assessments, standard interpretation of response rates referring to a one-time survey do not apply. ${ }^{15}$ We believe we achieved an adequate sample, as the majority of teams participated, the respondent demographics were proportional to the base population eligible to participate, and we received a similar number of cards on 
all months. Finally, although we were unable to account for clustering effects by individual respondents because response cards were anonymous, we adjusted for clustering effects by team.

Attending physicians may use our findings to adapt teaching techniques to appeal to specific training levels and to external pressures during teaching rounds. Focusing and investing time in teaching medical knowledge and clinical reasoning tailored to each level of learner is paramount on most days. However, days with a high workload may require

\section{References}

1. Roy B, Salanitro AH, Willett L, et al. Using cognitive mapping to identify attributes contributing to successful ward-attending rounds -- a resident and student perspective. J Gen Intern Med. 2010;25(S3):2.

2. Ben-Menachem T, Estrada C, Young MJ, et al. Balancing service and education: improving internal medicine residencies in the managed care era. Am J Med. $1996 ; 100(2): 224-229$.

3. Drolet BC, Bishop KD. Unintended consequences of duty hours regulation. Acad Med. 2012;87(6):680.

4. Drolet BC, Spalluto LB, Fischer SA. Residents' perspectives on ACGME regulation of supervision and duty hours--a national survey. N Engl J Med. 2010;363(23):e34.

5. Nasca TJ, Day SH, Amis ES, Jr. The new recommendations on duty hours from the ACGME Task Force. N Engl J Med. 2010;363(2):e3.

6. Shiffman S, Stone AA, Hufford MR. Ecological momentary assessment. Annu Rev Clin Psychol. 2008;4:1-32.

7. Moskowitz DS, Young SN. Ecological momentary assessment: what it is and why it is a method of the future in clinical psychopharmacology. J Psychiatry Neurosci. 2006;31(1):13-20.

8. Wingo MT, Halvorsen AJ, Beckman TJ, Johnson MG, Reed DA. Associations between attending physician workload, teaching effectiveness, and patient safety. emphasis on delegating clear, rational treatment plans, when learners are less receptive to traditional didactic methods.

Disclosure: An abstract based on the current analysis was presented at the Society of General Internal Medicine 34th Annual Meeting, April 2011, Phoenix, AZ. Dr. Brita Roy is supported by grant number K12HS023000 from the Agency for Healthcare Research and Quality. The authors have no conflicts to disclose. The opinions expressed in this article are those of the authors alone and do not reflect the views of the Department of Veterans Affairs or the Agency for Healthcare Research and Quality.

J Hosp Med. 2016;11(3):169-173.

9. Goldszmidt M, Faden L, Dornan T, van Merriënboer J, Bordage G, Lingard L. Attending physician variability: a model of four supervisory styles. Acad Med. 2015;90(11):1541-1546.

10. Kennedy TJ, Lingard L, Baker GR, Kitchen L, Regehr G. Clinical oversight: conceptualizing the relationship between supervision and safety. J Gen Intern Med. 2007;22(8):1080-1085.

11. Irby DM. How attending physicians make instructional decisions when conducting teaching rounds. Acad Med. 1992;67(10):630-638.

12. Kennedy TJ, Regehr G, Baker GR, Lingard LA. Progressive independence in clinical training: a tradition worth defending? Acad Med. 2005;80(10):S106-S111.

13. Tariq M, Motiwala A, Ali SU, Riaz M, Awan S, Akhter J. The learners' perspective on internal medicine ward rounds: a cross-sectional study. BMC Med Educ. 2010;10:53

14. Certain LK, Guarino AJ, Greenwald JL. Effective multilevel teaching techniques on attending rounds: a pilot survey and systematic review of the literature. Med Teach. 2011;33(12):e644-e650.

15. Stone AA, Shiffman S. Capturing momentary, self-report data: A proposal for reporting guidelines. Ann Behav Med. 2002;24(3):236-243. 